\title{
Increasing the accuracy of signal extraction by correcting the approximating function under conditions of a priori uncertainty
}

\author{
Ivan Nikishin, Vladimir Marchuk* ${ }^{*}$ Igor Shrayfel, Ilya Sadrtdinov \\ Don State Technical University, Institute of Service and Business (Branch) DSTU in Shakhty, Russia
}

\begin{abstract}
The paper discusses the issues of practical implementation of increasing the accuracy of signal extraction, which is achieved by eliminating the «flip» of the approximating function when dividing the measured process into intervals under conditions of a priori uncertainty about the signal function, which significantly increases the error of allocating a useful signal. The probability of a «flip» of the approximating function depends significantly on the variance of the additive noise and the sample length. The use of the proposed methods and their software implementation makes it possible to increase the accuracy of the useful signal extraction up to 30 percent in the absence of a priori information about the function of the measured process for complex signals and at least $20 \%$ for simpler ones. The use of the proposed methods will significantly increase the processing efficiency in the conditions of a priori uncertainty about the function of the measured process (useful signal) and the statistical characteristics of the additive noise components.
\end{abstract}

\section{Introduction}

When processing measurement results, a priori information about the function of the measured process is practically absent or extremely small, in accordance with [1] the original implementation is divided into quasi-stationary sections using Kendall statistics. This approach to the processing of measurement results is widely used in practice, in particular the method of RAZOTS [2-6] also uses interval partitioning using uniformly distributed random numbers. However, when approximating on each partition interval of the initial implementation by a polynomial of the second degree, it was noted in [2] that at a certain value of the partition interval and the value of the variance of the additive noise component, the effect of «flipping» of the approximating function occurs, which significantly increases the error in the extraction of the useful signal. It should be noted that even the use of the RAZOTS, method, based on the multiplication of the obtained estimates with their subsequent averaging, reduces its efficiency by more than $5 \%$ compared to the use for the approximation on each of the intervals of the partition by a polynomial of the first degree [2-4]. In [5] the probability of the appearance of the «flip» effect of the approximating function was investigated depending on the length of the approximation

\footnotetext{
${ }^{*}$ Corresponding author: $\underline{\text { marchuk@ @ssu. ru }}$
} 
interval and the variance of the additive noise component, which showed an inverse dependence on the length of the approximation interval and a direct dependence on the variance of the additive noise component. Since the splitting of the original implementation by any of the above methods (the RAZOTS method, Kendall statistics, etc.) always results in splitting intervals of different lengths, the «flip» effect can also be observed with relatively small variances of the additive noise component. In this regard, the problem of eliminating the noted drawback in approximation at each partition interval is very urgent, which will significantly improve the accuracy of approximation of the measured useful signal function.

\section{Methods and materials}

When solving this problem, it should be noted that the studies carried out in [8-20] showed that with an increase in the dispersion of the additive noise component, the probability of the appearance of a «flip» increases almost linearly. It should be noted that with a decrease in the sample size, the probability of a «flip» increases even with relatively small variances of the additive noise component [5]. So, for example, when the variance is $\sigma^{2}=0.5$ relative to the normalized value of the useful signal, the probability of a «flip» approaches 0.4 with a sample length $N=50$, and with a sample length of $N=10$ the probability of a «flip» approaches 0.6. Accordingly, in this case, the root-mean-square error of the extraction of the useful signal increases significantly [5-6]. In this regard, let us consider the issues of «переворота» the approximating function. The mirror transformation of the obtained estimate of the approximating function can be performed using the expressions given in [6] with respect to a certain straight line. The question about which line is the flip was studied in the work [6]. The results of the analysis showed that the mirror «flip» must be carried out relative to the straight line obtained using the LMS of the original implementation, while the error decreases 2.7 times compared to the straight line obtained by connecting the extreme points of the estimate by the approximating function.

To study the efficiency of mirroring, consider a simple case when the useful signal model is a parabolic function. With an increase in the variance of the additive noise, a «переворота», effect is observed, which will be replaced by a mirror image of the obtained estimate of the useful signal. The research results for this case are shown in Figure 1.

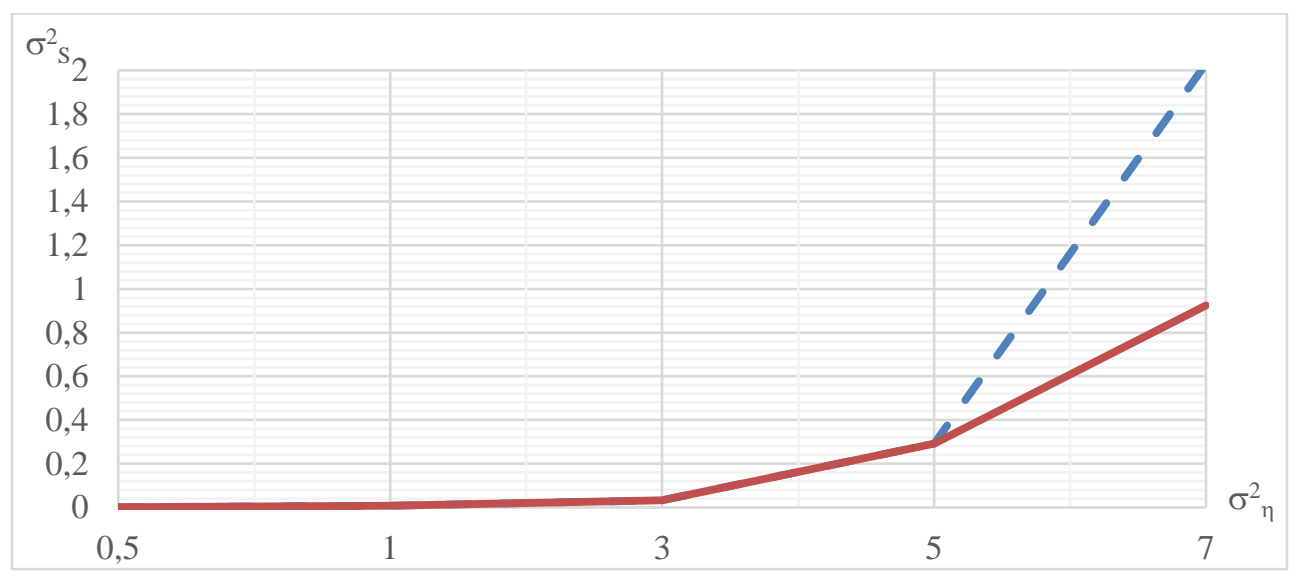

Fig. 1. Analysis of the efficiency of mirroring the approximating function in the presence of the «flip» effect 
As can be seen from the results presented in Figure 1, with a sufficiently large variance (the function is simple and the sample is 100 samples), a reversal is observed, which increases the processing error by more than 2 times (dashed line) after the variance is greater than 5. The use of a mirror flip increases the accuracy of processing, almost completely compensating for the error resulting from the «flip» of the approximating function (solid line). For a more detailed analysis of the efficiency of the mirror flip of the approximating function, let us consider the averaged error over 1000 realizations for a simple case - the useful signal model is a parabolic function. The research results are shown in Figure 2.

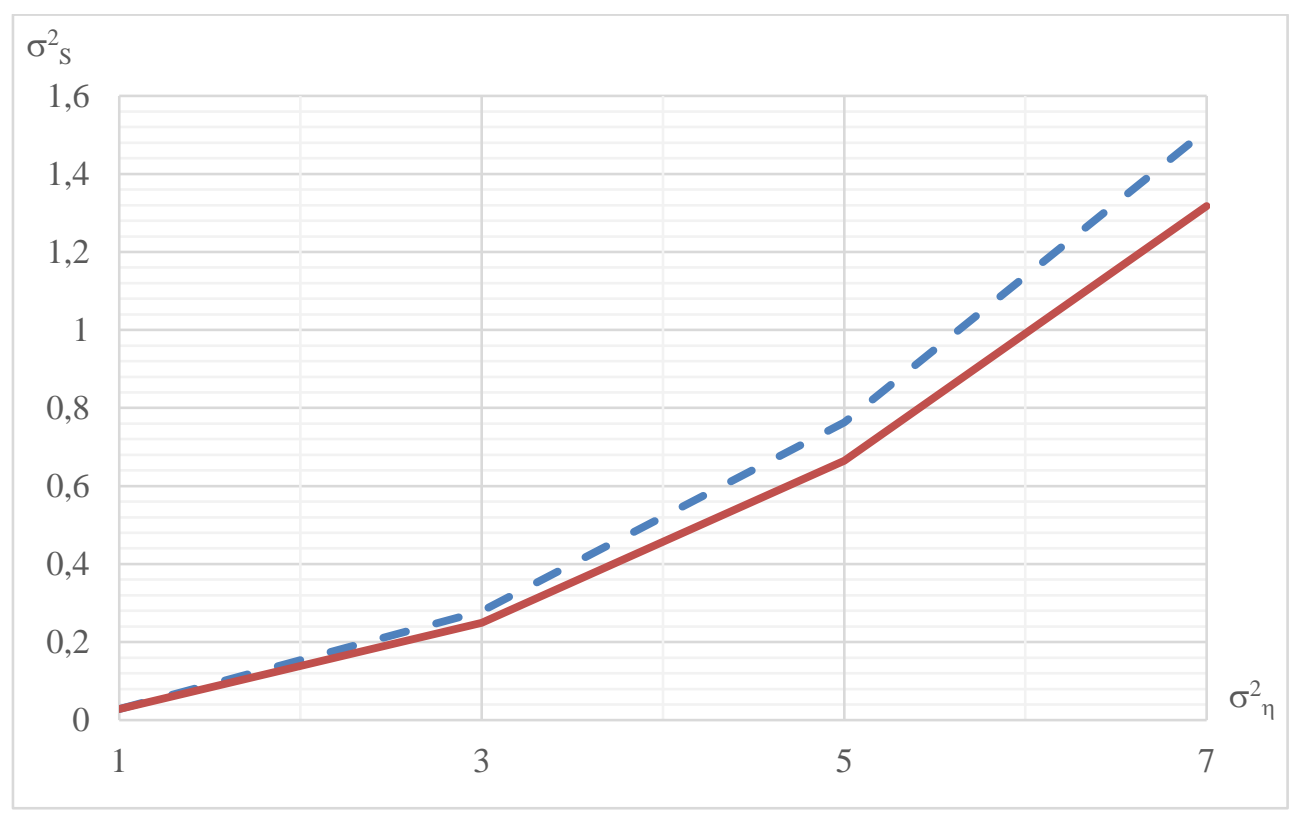

Fig.2. The dependence of the error in the extraction of the useful signal on the dispersion of the additive noise component in the presence of a «flip» (dotted line) and its replacement by a mirror (solid line) according to the proposed method

The analysis of the obtained results, presented in Figure 2, shows that the error in the extraction of a useful signal can be reduced by an average of $17 \%$. It should be noted that the probability of a flip is already observed for variances greater than 1 . However, in this case, the sample size is very large and of significant interest is the case when the sample is small and when the function cannot be approximated over the entire observation interval.

In this regard, the original sample was divided into three equal parts, on each of which an approximation was carried out with subsequent mirror transformation in the presence of the «flip» effect. The results of the studies are shown in Figure 3. Analysis of the results presented in Figure 3 allows us to conclude that the efficiency of using the mirror replacement of the approximating function in the presence of a «flip» increases, and the error in the selection of measurement results can be reduced by an average of $20 \%$.

From the analysis of the conducted studies, it can be concluded that when the original implementation is divided into shorter samples using Kendall statistics or based on the functioning of the RAZOTS method the probability of a «flip» increases and the use of the method of mirror replacement of the approximating function will be even more effective and a decrease in the extraction error of the useful functions by more than $20 \%$. 


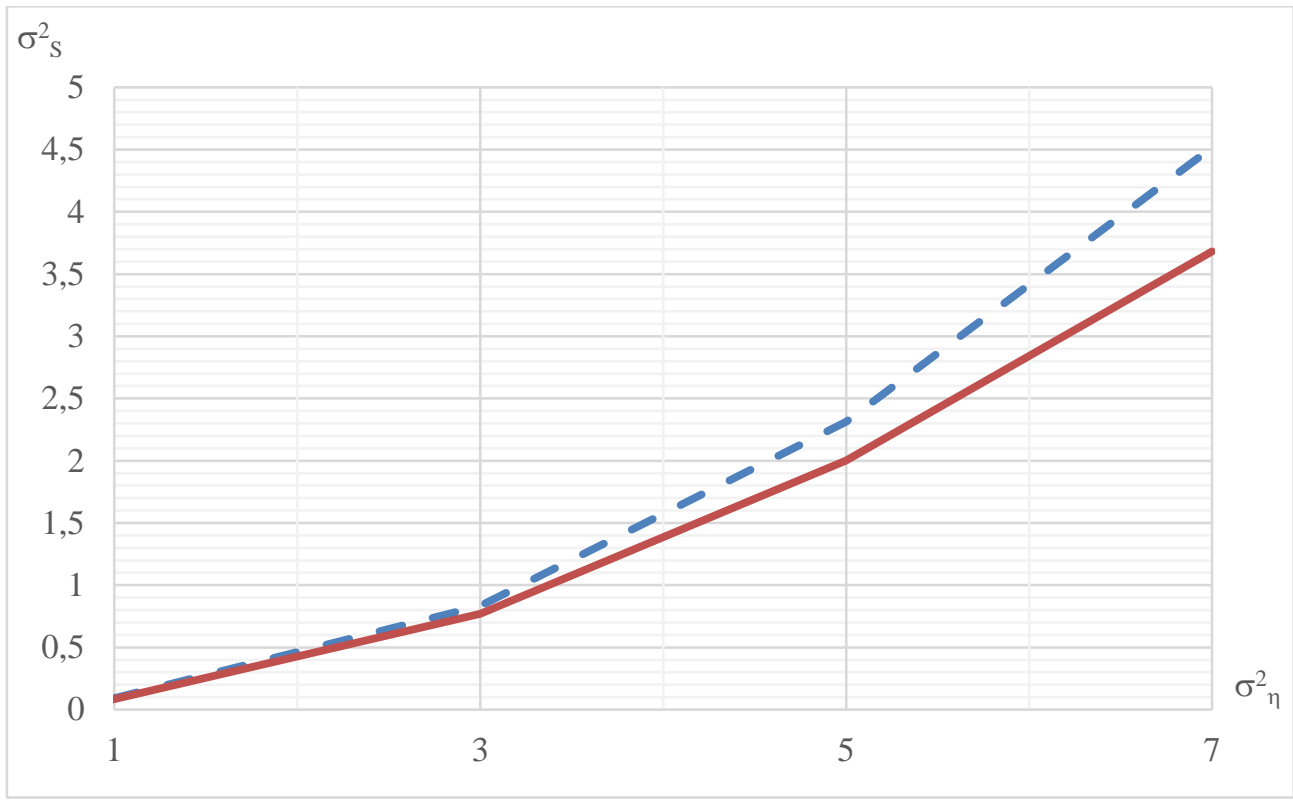

Fig.3. The dependence of the error in the extraction of the useful signal (the signal is divided into three equal intervals) on the dispersion of the additive noise component in the presence of a «flip» (dotted line) and its replacement by a mirror (solid line) according to the proposed method

\section{Results and discussion}

Thus, in this paper, the reason for the increase in the error of the useful signal extraction with an increase in the dispersion of the additive noise component is determined and a method for reducing it is proposed. The results of the computer simulation fully confirmed the analytical results and the effectiveness of the proposed replacement of the «flip» of the approximating function by its mirror image.

\section{Conclusion}

1. The presence of a «flip» when processing signals at some intervals of approximation significantly affects the error in the process of extraction of the useful signal.

2. With an increase in the variance of the additive noise component, the probability of the appearance of a «flip» grows almost linearly.

3. With the variance of the noise $\sigma^{2}=0.5$ relative to the normalized value of the useful signal, the probability of a «flip» approaches 0.4 with a sample length of $\mathrm{N}=50$, and with a sample length of $N=10$, the probability of a «flip» approaches 0.6 .

4. The results of the analysis show that the mirror «flip» must be carried out relative to the straight line obtained using the LMS of the original implementation, while the error decreases by 2.7 times.

5. The use of a mirror flip according to the obtained analytical expressions made it possible to reduce the error with a pure parabola by $17 \%$, and when it is divided into 3 parts - by $20 \%$. 
6. The use of the proposed technique to eliminate the «flip» in the method of multiplication of estimates (RAZOTS) allows to reduce the error by an average of $30 \%$, depending on the function of the useful signal.

\section{References}

1. S.M. Perevertkin, A.V. Kantor, N.F. Borodin, T.S. Shcherbakova. Moscow: Mashinostroenie (1977)

2. V.I. Marchuk, G.R. Saakyan, A.P. Ulanov. Method for identification of a trend by replicating the estimates of its single initial implementation (RAZOTS) and a device for its implementation. Patent No. 2207622, IPC G06F 17 / 18, applicant and patentee «South-Russian State University of Economics and Service» - No. 2000127308/09; declared 10/30/2000; publ. 06/27/2003 Bul. No. 11.

3. V.I. Marchuk, I.S. Shrayfel. Methods for isolating the useful component with a priori uncertainty and a limited amount of measurement results. Shakhty (2008)

4. V. Marchuk. Serbian journal of electrical engineering, 15, 3, 365-370 (2018)

5. V. Marchuk, D Chernyshov, I. Sadrtdinov, A. Minaev. E3S Web of Conferences, 104, 02003 (2019)

6. V. I. Marchuk, G.L. Hripkov, I.O. Nikishin, I.S. Shrayfel. IOP Conference Series: Materials Science and Engineering, 1029, 1, 012094 (2021)

7. T. N. Siraya. Measurement Techniques, 61, 1, 9-16 (2018)

8. G. N. Solopchenko. Measurement techniques, 59, 5, 476-484 (2016)

9. A. Chunovkina. Proceedings of 28-th National Scientific Symposium with international participation «METROLOGY and METROLOGY ASSURANCE 2018», 31-34 (2018)

10. J. Krejčí, D. Petri, M. Fedrizzi. IEEE Transactions on Instrumentation and Measurement, 66, 12, 3228-3236 (2017)

11. N.A. Serov, N.M. Kyaw, A.A. Lupachev, E.A. Elicheva, Y.S. Bekhtin, A.R. Petsinyarzh. XXIX International Scientific Symposium «Metrology and Metrology Assurance» (MMA), 1-4 (2019)

12. Y.S. Bekhtin, A.A. Lupachev, A.N. Serov, A.V. Kovalenko. Proceedings of 7th Mediterranean on Embedded Computing «MECO’2018», 4 (2018)

13. S. Bhattacharya, M. Henzinger, D. Nanongkai. Proceedings of the forty-eighth annual ACM symposium on Theory of Computing, 398-411 (2016)

14. V. Patel, G. Regts. SIAM Journal on Computing, 46, 6, 1893-1919 (2017)

15. Y. Gromov, Y.V. Minin, O.G. Ivanova, A.G. Divin, S.L. Majeed. Journal of Theoretical and Applied Information Technology, 96, 23, 7892-7903 (2018)

16. R. Stoean, C. Stoean, M. Atencia, R. Rodríguez-Labrada, G. Joya. Mathematics, 8, 7, 1078 (2020)

17. S. Roy, S. Baldi, L. M. Fridman. Automatica, 111, 108650 (2020)

18. A.P. Trifonov, Y.E. Korchagin, K.D. Titov. Technical Physics, 63, 8, 1199-1204 (2018)

19. P. Chahuara, F. Portet, M. Vacher. Expert Systems with Applications, 75, 63-79 (2017)

20. E.V. Eremin. Measurement Techniques, 63, 1, 15-22 (2020) 